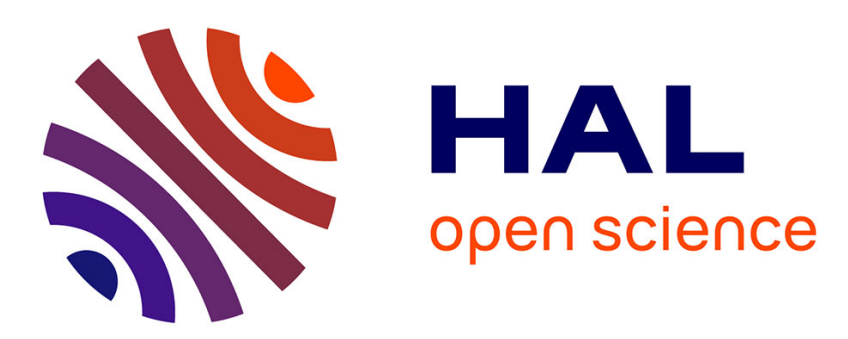

\title{
Jeux d'echelle et enjeux : dynamiques identitaires des ceremonies processionnelles en Birmanie bouddhique
}

François Robinne

\section{To cite this version:}

François Robinne. Jeux d'echelle et enjeux: dynamiques identitaires des ceremonies processionnelles en Birmanie bouddhique. Aséanie, Sciences humaines en Asie du Sud-Est, 2008, 22, pp.121-150. halshs-01866764

\section{HAL Id: halshs-01866764 \\ https://shs.hal.science/halshs-01866764}

Submitted on 3 Sep 2018

HAL is a multi-disciplinary open access archive for the deposit and dissemination of scientific research documents, whether they are published or not. The documents may come from teaching and research institutions in France or abroad, or from public or private research centers.
L'archive ouverte pluridisciplinaire HAL, est destinée au dépôt et à la diffusion de documents scientifiques de niveau recherche, publiés ou non, émanant des établissements d'enseignement et de recherche français ou étrangers, des laboratoires publics ou privés. 


\section{Jeux d'échelle et enjeux:}

\section{Dynamiques identitaires des cérémonies processionnelles en Birmanie bouddhique}

François Robinne

\section{Dynamique des échanges et domination du paysage social}

I es relations interethniques ${ }^{1}$ autour du lac Inlé et dans la plaine rizicole tions symboliques apportées à deux rituels processionnels bouddhiques. Bien qu'ils se déroulent l'un et l'autre dans deux régions de l'état shan, aucun lien ne réunit les deux rituels. En revanche, l'usage spécifique qui est fait dans les deux cas de la valeur commune qu'est le bouddhisme - avec son lot de transformations et de variantes apportées ici et là aux formes rituelles - est partie constitutive d'un processus dont les enjeux interfèrent à un double niveau d'échelle. Au niveau local, les aménagements successifs des deux rituels s'inscrivent dans un processus de domination du paysage social - au sens de complexe multiethnique -, tandis qu'au niveau du

1. L'expression « relations interethniques » nécessite quelques précisions terminologiques. Elle renvoie à quatre notions complémentaires, dont les définitions suivantes ont été retenues par souci de convention: (1) «multiethnicité » définit une situation de diversité linguistique et culturelle dans une région donnée; (2) « interethnicité » fait référence aux relations entre groupes limitrophes, avec la part de hiérarchie inhérente à la dynamique relationnelle, ou entre minorités et pouvoir central, que ce dernier soit ou non associé à une catégorie ethnique; (3) «pluriethnicité » s'applique à la revendication par un individu de plusieurs identités, au contraire de (4) " transethnicité ", qui caractérise une situation où la dynamique des réseaux d'échanges quels qu'ils soient - économiques, religieux, matrimoniaux, etc. - relègue au second plan le référent ethnique au profit de la notion de partenariat (Robinne 2007, 283-297; Robinne et Sadan 2007, 306 ; Robinne 2008, 136-139). 
de Thibaw), la famille austro-asiatique (sous-groupes danaw à Inlé et palaung à Thibaw), la famille karen (sous-groupe pao dans l'une et l'autre régions ${ }^{2}$ ) et la famille tai-kadai (sous-groupes shan et tai établis à Thibaw dans les plaines rizicoles, mais désormais absents du paysage social à Inlé). Un tel découpage n'est représentatif ni des rapports de force sous-jacents ni de l'imbrication effective issue des réseaux d'échanges économiques, religieux ou matrimoniaux; la dynamique des réseaux et leurs intérêts parfois contraires tantôt transcendent, tantôt tendent à réifier le référent ethnique du processus identitaire.

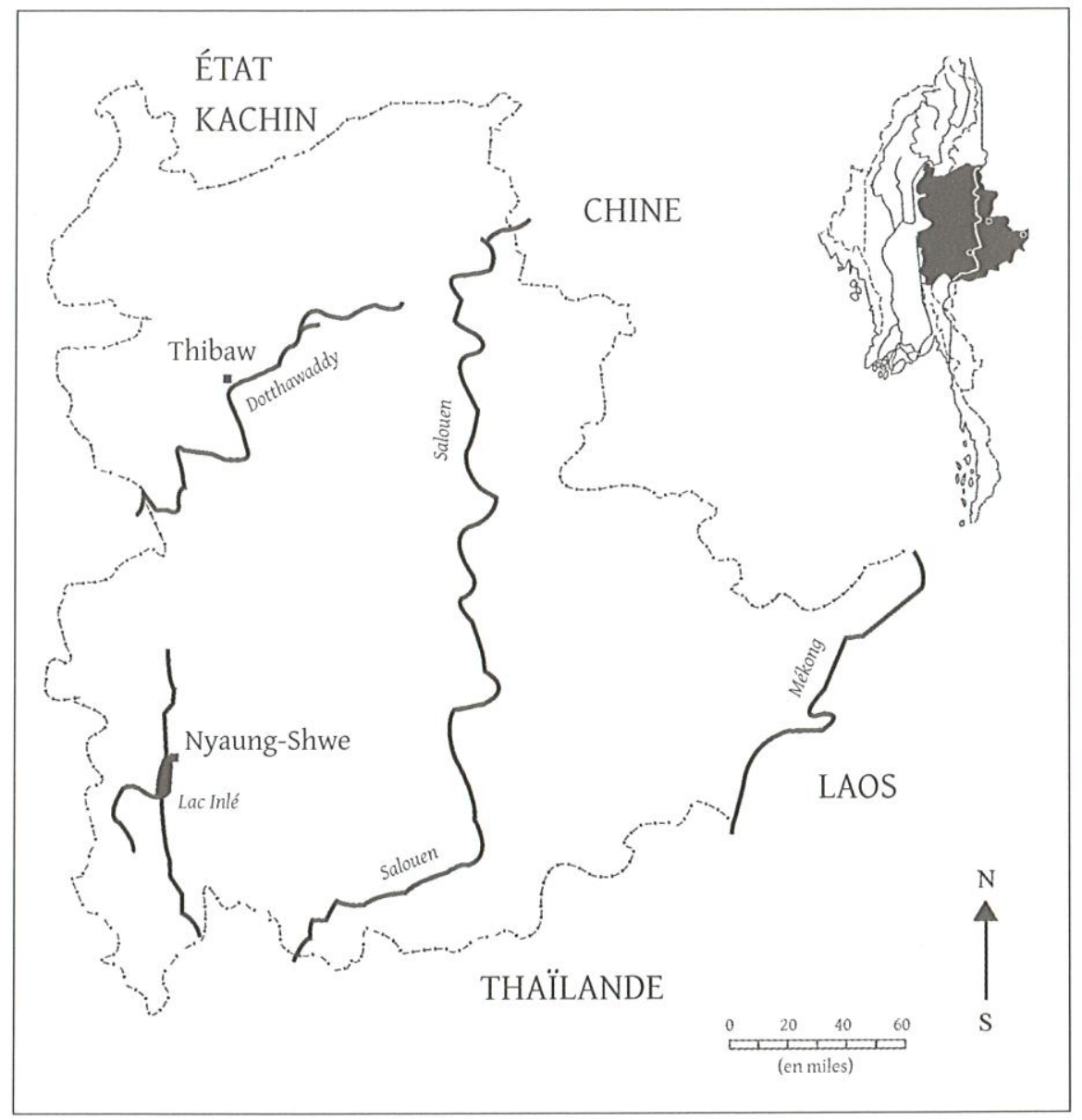

Fig. 1. Situation de Thibaw et de Nyaung-Shwé sur le lac Inlé, État shan de Birmanie

2. Bien qu'ils appartiennent à la même famille linguistique, les Pao ne se reconnaissent pas en tant que Karen. Ceux-ci sont globalement assimilés à des chrétiens ou à des communistes, deux critères d'autant plus rédhibitoires qu'ils sont parfois associés. 
La transhumance de buffles et les réseaux caravaniers à dos de mules participent non seulement au dynamisme des réseaux interethniques, mais ils interfèrent également dans le rapport hiérarchique entre les hautes terres et les basses terres. De nos jours encore, dans la région de Thibaw, dans le nord de l'État shan, les Palaung conduisent à dos de mules leur production de thé vert et de thé mariné jusqu'au marché de la capitale régionale; ils remontent chargés d'huile, de sel, de cigares, de riz blanchi. Ce type de commerce est suffisamment important pour avoir été attesté par les administrateurs britanniques du début du xx siècle, sous forme notamment de caravanes de mules conduisant les productions de thé vers les marchés des basses terres (vers la «Birmanie » disent, de façon significative, les Palaung comme, de manière générale, les populations des hautes terres de Birmanie), avant de repartir dans les collines avec un chargement de vêtements, d'images de Bouddha et autres objets de culte, des bols vernissés, du tabac et le nécessaire à chiquer, etc. (Milne 1924,222). Tout laisse à penser que la régularité de tels échanges est contemporaine de l'introduction, simultanée selon la légende, du thé et du bouddhisme dans la région, c'est-à-dire vers le XIII ${ }^{e}$ siècle. De manière sans doute tout aussi ancienne, des négociants chinois établis en ville se rendent également dans les montagnes: ils y achètent des mules pour les réseaux caravaniers et des cargaisons de thé destinées aujourd'hui au marché de Mandalay, dans le centre de la Birmanie. Quant aux riziculteurs shan, du fait même de leur spécialisation dans le domaine de la riziculture inondée, ils sont en grande mesure dépendants des montagnards pour l'acquisition de bêtes de trait dont la demande se fait pressante deux fois l'an, avant les labours et après la récolte ; les buffles sont loués aux Palaung qui les mènent vers les basses terres: la location des bestiaux le temps d'une saison est de deux sacs de riz par animal ${ }^{4}$.

Dans son travail précurseur, Pierre Gourou a mis en évidence l'incompatibilité entre riziculture inondée et élevage, avec la nécessité de distinguer entre la possession de deux ou trois têtes de bétail nécessaires aux travaux agricoles et la propriété de troupeaux tournés vers la reproduction, la production de viande et celle de produits laitiers (Gourou 1972, 114-120). Dans le même sens, j'ai pour ma part contribué à montrer que les sentiers tracés par la transhumance des buffles et les réseaux caravaniers à dos de mule attestent du lien de complémentarité entre la riziculture inondée des basses terres et l'élevage de bestiaux concentré en partie dans les hautes terres (Robinne 1998, 2000a et 2000b). La propriété des bêtes de trait et de labour étant essentiellement entre les mains des montagnards, l'échange de bestiaux tend, là encore, à inverser le rapport de dépendance au détriment des riziculteurs.

4. Pour le thème de l'échange des buffles et ses implications dans les échanges interethniques, voir Robinne 1998 et 2000a. 
tion entre différents systèmes politiques « le langage rituel commun » donnant au paysage social ainsi défini sa véritable cohérence.

Lorsque le coup d'État birman de 1962 mit un terme aussi subit qu'irréversible à l'hégémonie des administrations hiérarchisées calquées pour la plupart sur le modèle shan, le remodelage des relations interethniques s'imposa, de même que la recomposition de nouvelles hiérarchies. La suprématie séculaire des administrations shan sur leurs voisins immédiats ayant été anéantie du jour au lendemain, le paysage social fut assujetti au contrôle direct des autorités birmanes. Au niveau local, le rapport passa de la domination d'un groupe sur plusieurs autres inféodés à un système de type égalitaire où l'économique régula pour l'essentiel la dynamique des échanges.

Le pouvoir politique ayant été désintégré, le bouddhisme s'affirma - dans ces deux régions, comme ailleurs dans l'État shan - le fédérateur commun aux différentes forces en présence. Le bouddhisme theravādin et la dynamique des réseaux à laquelle il participe parfois très directement constituent là encore un élément de comparaison entre les régions d'Inlé et de Thibaw; sa pratique est consubstantielle à l'ensemble des groupes considérés ici, à l'exception des Lisu de confession chrétienne dont la présence, on le verra, est marginale dans la région de Thibaw. Quelle que soit l'appartenance linguistique et quelle que soit l'aire d'implantation privilégiée, dans les plaines alluviales ou sur les versants des montagnes environnantes, le bouddhisme ne constitue par conséquent pas en soi un domaine réservé à l'un ou l'autre des groupes en contact, pas plus qu'il ne positionne a priori tel ou tel groupe en position de force à l'égard de ses voisins. Ajoutons que, pas plus que d'autres sphères du social, ce fédérateur commun qu'est le bouddhisme ne saurait réduire les particularismes et les individualités des voies dont il est lui-même empreint dans les formes rituelles; il ouvre bien au contraire un univers de possibles beaucoup plus qu'il ne fixe des règles strictes et exclusives (Robinne 2000a et 2000b). En cela le bouddhisme participe tout à la fois de la diversité des pratiques socioculturelles et du dynamisme des échanges.

À maints égards, les deux régions d'Inlé et de Thibaw sont comparables, tant du point de vue de la diversité de leur peuplement que de la vitalité des réseaux interethniques ou encore de la prégnance du bouddhisme. La question qui se pose, et qui en l'état reste en suspens, est de savoir si la tendance amorcée ces dernières années d'une prééminence économique au profit des montagnards s'accompagnera ou non d'un transfert simultané d'une dimension symbolique. Quoi qu'il en soit, la présence de cérémonies processionnelles bouddhiques contribue dans les deux régions à donner à l'ensemble multiethnique sa cohérence. La similitude des deux paysages sociaux s'impose donc, à cette réserve près que les processions d'Inlé et de Thibaw ont connu des évolutions inverses à partir du coup d'État birman de 1962. 
et le Bouddha futur? ${ }^{7}$ À chacune d'elles correspond un point cardinal qui détermine leur position sur l'autel octogonal. Leurs dimensions réduites contrastent avec les images inamovibles qui peuvent se trouver à la base de certains stūpas. Elles sont sorties une fois l'an du hall d'accueil situé en aval du lac, où elles reposent habituellement, pour être emportées tout au long d'une vaste circumambulation dans un nombre toujours croissant de villages étapes situés à la périphérie du lac. Le cortège se compose d'une embarcation à figure d'oiseau mythique tirée par une succession de longues pirogues reliées entre elles par un filin. À chaque escale, les images pieuses sont transportées dans les monastères ou halls d'accueil selon un ordre immuable: l'image située à l'est tout d'abord, puis celle du sud, puis celle du nord, puis celle de l'ouest; l'image centrale, celle représentant Kakusandha, le premier des trois Bouddha antérieurs, est laissée dans le hall d'accueil principal. À Inlé, on sait que les sawbwa de Nyaung-Shwé allèrent dans un premier temps à la rencontre des effigies pour les accompagner eux-mêmes jusqu'au palais en amont du lac; ce dernier fut par la suite intégré au parcours, dont il constitua une étape parmi d'autres, à cette différence près que les images y séjournaient trois nuits au lieu d'une seule ailleurs (Robinne 2000a).

Les adaptations apportées peu à peu à la cérémonie processionnelle qui parcourt le lac Inlé vingt-six jours durant - contre cinq auparavant - se sont échelonnées dans le temps. Elles furent sanctionnées immanquablement par une manifestation des images pieuses à l'origine de la circumnavigation: tempêtes, accidents et autres événements sont interprétés comme autant de représailles surnaturelles qui ponctuèrent dans un rapport de cause à effet chaque élargissement de la cérémonie. Le nombre des figurines emportées s'en trouva lui-même modifié. Faisant suite à un chavirage, il fut décidé que quatre seulement des images participeraient désormais à la circumambulation, la cinquième devant rester en permanence sur son piédestal. Dans le même temps, le parcours désordonné de la cérémonie d'une rive à l'autre du lac fut réorganisé pour former une déambulation rituelle de plus en plus étendue, parcourant le lac dans le sens de la course du soleil à l'instar de la circumambulation (pradakșinā) effectuée par les pèlerins autour d'une pagode: c'est en gardant à main droite le hall d'accueil, dans lequel se trouve en permanence la cinquième image, que la circumambulation des quatre autres représentations du Bouddha est effectuée chaque année.

D'un point de vue sociologique, plusieurs raisons peuvent être avancées à ces transformations : dans leur rapport à la surnature et au clergé, les changements succes-

7. Les cinq images sont positionnées sur un autel octogonal à l'étage supérieur d'un hall d'accueil sous lequel se trouve un marché permanent. Le hall est ouvert à longueur d'année, au contraire des halls d'accueil provisoires des villages étapes qui ne sont ouverts au public qu'une fois l'an, à l'occasion de la cérémonie processionnelle. Ces cinq images représentent: Kakusandha au centre, Konaghamāna au sud-est, Kāsāpa au sud-ouest, Gotama au nord-ouest, Aghuimettawya au nord-est. 
qui, sur le plan sociologique, déborde le seul parcours suivi par les images de Bouddha. Les villageois des montagnes environnantes convergent vers chacun des villages étapes; ils s'en retournent riches d'un acte méritoire supplémentaire, dont la retombée en termes karmiques est censée rejaillir sur chaque pèlerin et s'étendre à l'entourage du donateur. À un jeu d'échelle supérieur, le pouvoir mystique9 émanant de chaque image à qui il a été rendu hommage est étendu à l'ensemble de la région. La configuration paradigmatique des cinq images d'Inlé est censée renforcer le pouvoir de cette entité indissociable étendue à la région. Faisant suite à l'affaiblissement puis à la chute des administrations shan sous l'autorité desquelles la région fut placée des décennies durant, celle-ci s'est forgé une nouvelle cohérence en identifiant le paysage à ce modèle de quatre points autour d'un centre. Les quatre extrémités cardinales du lac en sont peu à peu venues à faire l'objet d'un séjour prolongé des images une fois l'an, tandis que le hall d'accueil central où siègent habituellement les cinq images s'est imposé de façon concomitante comme le pôle autour duquel s'organise l'espace régional. Dans le même temps où la circumambulation en vint à dessiner symboliquement le contour lacustre, son influence s'affirma sur l'ensemble du paysage social par l'empreinte architecturale qu'elle y dessine et par la dynamique des échanges rituels et économiques qu'elle régule pour partie, populations des hautes et des basses terres confondues. Le confinement relatif dans lequel se trouvent certains villages et la discontinuité des fêtes de pagode sont désormais corrigés par la solidarité que génère la cérémonie processionnelle.

La procession cérémonielle fait plus que générer l'unité et donner à la diversité du paysage social sa cohérence intrinsèque; elle contribue également à instaurer sur le plan symbolique une hiérarchie entre les groupes. Sans revenir dans le détail sur un processus analysé par ailleurs (Robinne 2000a), rappelons seulement que la fête de pagode des origines se trouvait vraisemblablement en territoire pao, dans un village de montagne situé à l'ouest du lac. Les Shan s'approprièrent par la suite les cinq images pieuses: dans le même temps où ils créèrent la cérémonie processionnelle, les différentes responsabilités rituelles - porteurs de parasol, rameurs, barreurs, musiciens, etc. - furent concentrées entre les mains des seuls Shan, dont certaines familles devinrent les détentrices héréditaires. En s'appropriant à leur tour la cérémonie processionnelle, les Intha mirent partiellement un terme à la transmission héréditaire des fonctions rituelles; celles-ci sont désormais attribuées en priorité à des servants de pagode choisis dans leurs rangs.

Les Intha ont donc donné à leur suprématie économique un prolongement symbolique, tout comme l'avaient fait auparavant les sawbwa shan, dont la mainmise sur le rituel processionnel confortait leur domination politique dans la région. L'appropriation du rituel sur une base ethnique constitue en quelque sorte l'aboutissement de la hiérarchie des relations interethniques, hiérarchie dont l'intensité

9. Tan Khui: en translittération, prononcé « dago ». 
également accueillir les images de Bouddha lors de l'escale de mi-parcours effectuée dans la ville de Nyaung-Shwé, en amont du lac: les images y restent trois jours, durant lesquels sont organisées des courses de pirogues. La présence des autorités birmanes en provenance de Rangoun fit la une du quotidien pro-gouvernemental The New Light of Myanmar en date du cinquième jour de la lune croissante du mois de thadingyut de l'année birmane 1356 (24 octobre 1994):

Vice-Chairman of the State Law and Order Restoration Council Deputy Commander-in-Chief of Defence Services Commander-in-Chief (Army) General Maung Aye, accompanied by Secretary-1 of the State Law and Order Restoration Council Lt-Gen Khin Nyunt [...], ministers, senior Tatmadaw officers, local populace and pilgrims throughout the nation paid homage to the Buddha images being conveyed on board the float back to the pagoda [...]

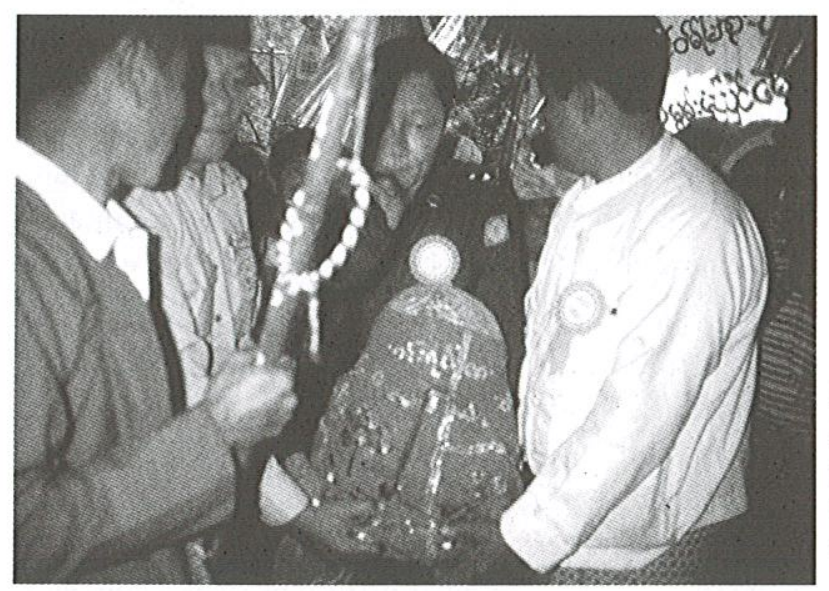

Fig. 2. Transport par un membre de la junte de l'une des quatre images du karaweik vers un hall d'accueil (Inlé, 1994)

À défaut d'avoir réussi à déplacer les images d'Inlé vers Rangoun, comme la rumeur leur en a prêté l'intention au milieu des années 1990, les plus hauts dirigeants régionaux et nationaux font depuis lors le déplacement vers le lac une fois l'an afin de présider la cérémonie. C'est de fait dans un contexte politique particulièrement tendu qu'il convient de replacer de tout temps la circumnavigation autour du lac Inlé. Dans ses élargissements successifs comme dans l'évolution de ses composantes ethniques, le rituel processionnel est réactif à l'histoire du pays.

Un schéma comparable caractérise la procession de Thibaw, plus au nord dans le même État shan. Jusqu'au coup d'État de 1962, la cérémonie de Thibaw constitua elle aussi l'assise symbolique de l'administration des sawbwa shan, qui contribuèrent pour beaucoup à son expansion. 
de quatre images de Bouddha promenées à travers la ville et dans ses environs. La présence de ces images est associée aux premiers rois birmans fondateurs de Pagan. La chronique de Thibaw - comme celle de Inlé-Nyaung-Shwé - rapporte que le roi birman Narapatisithu (1174-1211 AD, souvent confondu avec son grand-père Alaungsithu) y déposa les quatre images au prétexte que « les bouddhistes y étaient très nombreux comme partout ailleurs dans l'État shan et [que] l'amitié entre les deux peuples [était] aussi longue que le règne du Bouddha » (Rb-3 s. d., 11 ${ }^{11}$ ). Tout comme les images d'Inlé, celles de Thibaw sont taillées dans de l'Hopea elferi, un bois connu sous le nom de «l'arbre dhamma »; selon le fonds légendaire, l'introduction de cet arbre en Birmanie est associée à l'arrivée de Gautama Bouddha dans le pays (Rb-3 s. d., 13). Ces quatre images, ou « joyaux » dans la terminologie locale, font chacune face à un point cardinal ${ }^{12}$; la position centrale y est symbolisée, non pas par une cinquième image ${ }^{13}$ comme à Inlé, mais par la pagode Bokyaw érigée à l'endroit même où le Bouddha serait apparu dans l'une de ses vies antérieures sous forme de roi des cochons: c'est autour d'elle que se dressent les quatre images à l'origine de la cérémonie processionnelle et que déambulent les pèlerins en gardant la fondation pieuse à main droite.

En l'état actuel des connaissances, rien ne permet de dater avec certitude ni même de reconstituer avec précision les phases successives de transformation ayant marqué le passage d'une simple fête de pagode qu'était vraisemblablement la cérémonie de Thibaw à une cérémonie processionnelle. Néanmoins, plusieurs témoignages recueillis sur place permettent de reconstituer dans ses grandes lignes le parcours suivi par les images. Après avoir été transférées de la pagode Bokyaw jusqu'au palais du sawbwa shan - ou l'un des monastères rattachés au palais - celui-ci devint le hall d'accueil permanent des quatre images de Thibaw. Le palais constitua dès lors le point de départ et d'arrivée de la cérémonie processionnelle initiée, comme à Inlé, par les sawbwa shan. Dans un premier temps, les images étaient conduites du palais jusqu'à la rivière; de là on leur faisait remonter le cours de la Dotthawaddy sur une barge sacrée à figure d'oiseau mythique identique à celle employée sur le lac Inlé, par référence dans les deux cas au véhicule attaché dans l'imaginaire collectif à la divinité Sakra et aux rois birmans; les images étaient ensuite conduites à dos d'éléphants à travers la ville jusqu'à la pagode Mahamuni au nord-ouest de Thibaw; la procession effectuait alors plusieurs escales dans des villages étapes jusqu'à la pagode Bokyaw au sud de la ville, d'où les images étaient ramenées au palais.

11. Les références « $\mathrm{Rb}$ » suivies d'un chiffre renvoient dans la bibliographie ci-dessous aux références en birman.

12. Les quatre images de Thibaw sont dénommées et orientées comme suit: ratanā nya oń pour le Bouddha orienté au sud, ratanā zan oń - appelé aussi la proue de l'embarcation royale par référence au véhicule de Sakra - pour le Bouddha orienté à l'ouest, ratanā ran oń pour le Bouddha orienté au nord, ratanā mān oń pour le Bouddha orienté à l'est.

13. Selon certains informateurs, la pagode Bokyaw serait construite sur une cinquième image. 
un long périple de deux mois conduisant les images bien au-delà de l'État shan, dans les quatre divisions administratives de Mandalay, de Pégu, de Rangoun et de Magwé. Les sommes ainsi réunies financèrent la restauration de la pagode Bokyaw telle qu'on peut la découvrir actuellement, les images jusqu'alors amovibles étant fixées sur un piédestal aux quatre points cardinaux. Par un développement inverse à celui ayant prévalu dans un premier temps, la cérémonie processionnelle retrouva ainsi sa structure première d'un rituel sédentaire.

La sédentarisation de la cérémonie ainsi opérée offrait au pouvoir central l'avantage de briser la portée du lien communautaire que ce rituel véhiculait. En effet, dès lors, la responsabilité de l'organisation du rituel n'était plus localement l'apanage d'une communauté donnée: pas plus les sawbwa shan (ou leurs représentants depuis leur éviction) que toute autre entité ethnique politiquement organisée - tels les Palaung, les Pao ou encore les Lisu présents dans la région - n'étaient susceptibles d'occuper le leadership laissé vacant. Par ailleurs, dès lors que la convergence vers une seule et unique fête de pagode se trouvait substituée à la dynamique relationnelle intervillageoise, la sédentarisation du rituel processionnel eut pour effet de réduire la dynamique régionale des mouvements de population: l'exemple d'Inlé montre que chaque village d'accueil de la cérémonie processionnelle est partie constitutive d'un réseau de partenariat et de répartition des tâches - tant symboliques qu'économiques - étendu à un groupement de villages. La sédentarisation du rituel processionnel de Bokyaw permit au pouvoir central birman et à ses représentants locaux de sélectionner les élites locales parmi ses représentants et les servants de pagodes (trustees) qu'elle contrôle et de centraliser les réseaux d'échanges autour d'un marché unique en lieu et place du réseau généré par le cycle des cinq marchés tournants qui structurent encore les échanges dans plusieurs régions de l'État shan, au nombre desquelles la région Inlé (Robinne 1998 et 2000a). En même temps qu'il retrouvait sa configuration originelle de fête de pagode, le rituel perdait ainsi la dimension de vecteur des échanges et de la hiérarchie interethnique contenue dans la cérémonie processionnelle.

L'ampleur des réseaux de pèlerins s'en trouva, en effet, considérablement atténuée. La cérémonie d'ouverture est aujourd'hui réservée à quelques élites. Seuls des bonzes en provenance d'une trentaine de monastères, suivis des autorités militaires régionales, des servants de pagode, ainsi que d'une troupe folklorique de Palaung, de Shan et de Pao vêtus de leurs vêtements traditionnels, sont désormais autorisés à effectuer dans cet ordre la déambulation rituelle autour de la pagode. Ce n'est que les jours suivants que les villageois des hautes terres comme des basses terres sont autorisés à se rendre sur les lieux, où un imposant marché et des troupes théâtrales accompagnent le rituel bouddhique. Les autorités locales canalisent ainsi l'événement annuel majeur dont elles déplacent le jeu d'échelle en même temps que les enjeux: tandis que la cérémonie processionnelle imposait sa hiérarchie au bénéfice d'une administration shan dont l'assise symbolique reposait sur une dynamique partici- 
reproduisant en cela un processus assez courant chez nombre de montagnards d'Assam, de Birmanie et du Laos (Condominas 1980). Rien d'étonnant par conséquent à ce que la junte birmane ait également mis un terme aux ambitions potentielles des chefferies palaung dans la région.

Ce sont par conséquent des raisons essentiellement externes, liées au pouvoir central birman, qui sont à l'origine du blocage observé à Thibaw. Les facteurs internes qui tiennent à la diversité linguistique, au système éclaté des chefferies villageoises et aux patrilignages exogames sur lesquels reposait l'organisation sociale des Palaung ne sauraient en être la cause. En effet, la dynamique des réseaux d'échanges interethniques dresse pour ainsi dire mécaniquement un pont entre les frontières culturelles, tout comme le langage rituel commun que constitue le bouddhisme dépasse à lui seul la ligne de clivage ethnolinguistique. À Thibaw comme à Inlé, l'enjeu de gestion sociale du paysage inhérent aux cérémonies processionnelles tend à s'effacer derrière le contrôle exercé par les autorités birmanes. La création concomitante d'une parade sur le lac Kandawkyi à Rangoun participe de ce processus de birmanisation des rituels locaux dont elle tend à s'attribuer la dimension symbolique après qu'en ont été extraits leurs enjeux politiques.

\section{Vers une orientation corporatiste des cérémonies processionnelles}

Bien qu'ayant suivi deux évolutions contraires après les événements de 1962, les cérémonies autour du lac Inlé et de la région Thibaw eurent dès lors en commun d'avoir fait l'objet de la plus grande attention de la part du pouvoir central. En quête de légitimité, la junte birmane fit plus qu'exercer un contrôle strict sur les deux cérémonies processionnelles. C'est sous l'influence des autorités birmanes qu'elles connurent dans le même temps un cheminement inverse; les événements de 1988 précipitèrent la tendance en renforçant dans un cas l'expansion dans l'espace et dans le temps du rituel d'Inlé, en provoquant dans l'autre le démantèlement de la procession réduite à son expression minimaliste de fête de pagode pour le rituel de Thibaw. Mais les développements contraires apportés aux deux cérémonies n'en ont pas moins eu des effets similaires, à savoir: la tendance à la neutralisation de ces rituels par les dimensions extrêmes qu'ils prennent et, à travers eux, la tendance à la neutralisation du processus d'appropriation du paysage social désormais accaparé par le seul pouvoir central.

Dépossédée de son cadre politique que constituaient les administrations hiérarchisées des sawbwa shan, la toile de fond qu'est le bouddhisme subit les conséquences du dédoublement opéré localement entre le politique et le religieux. L'extension de la cérémonie processionnelle d'Inlé connaît actuellement des limites au-delà de laquelle elle perdrait le rôle d'opérateur commun qu'elle tient encore; inversement, la réduction du rituel processionnel de Thibaw à une fête de pagode connaît des limites en deçà desquelles le bouddhisme ne serait plus le fédérateur commun dont est emprunt le 


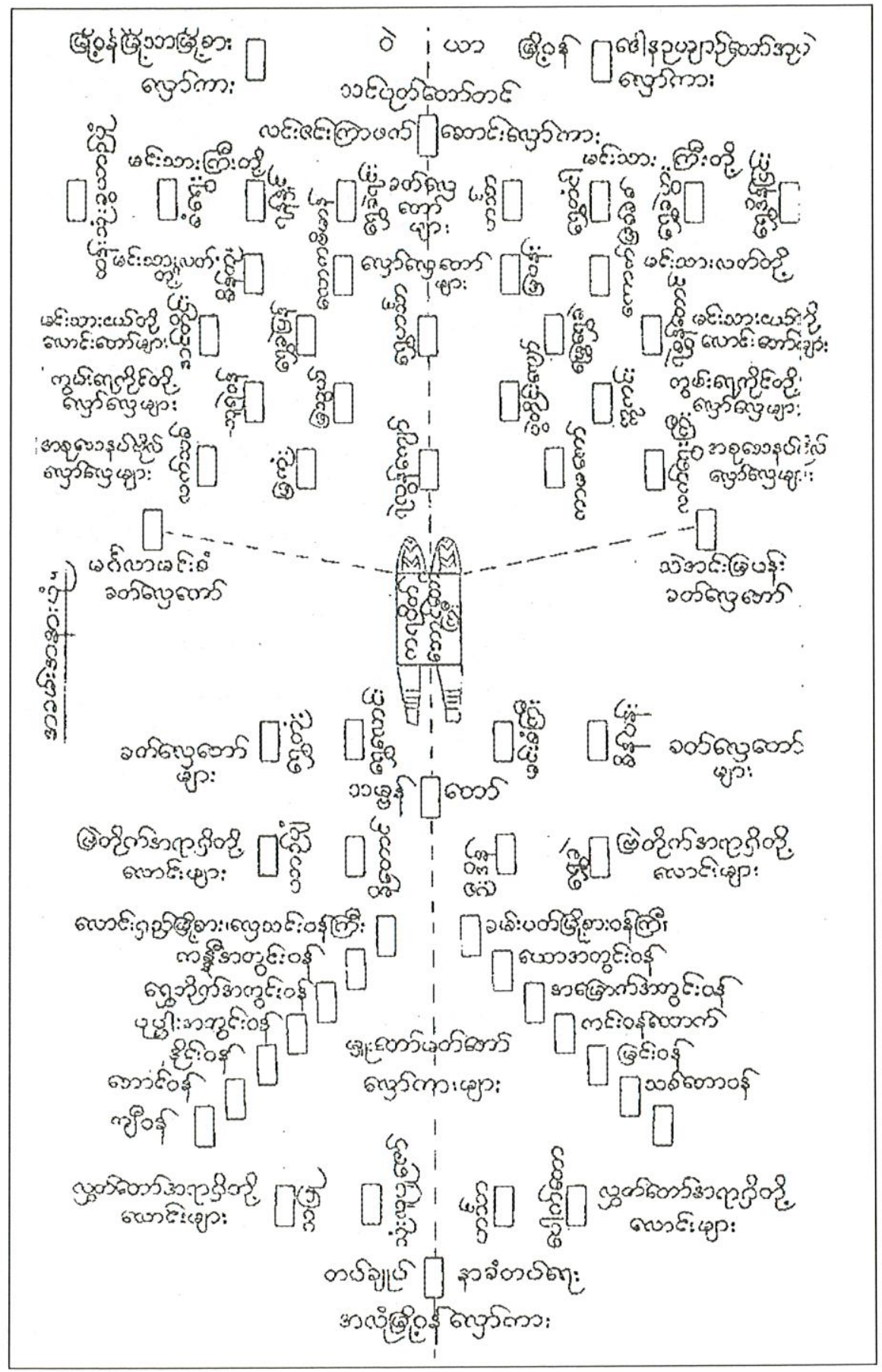

Fig. 4. Parade royale effectuée le douzième jour de la lune croissante du mois de nayon de l'année sakkarāj 1236 (1874 AD) à Mandalay (Rb-1:15) 
d'un fond historico-légendaire plus ancien, lié au roi birman Alaungsithu. Ce roi, qui régna à Pagan au XII ${ }^{\mathrm{e}}$ siècle, est connu pour avoir parcouru tout le pays sur une embarcation à figure d'oiseau mythique, emportant avec lui des figurines de Bouddha que la divinité supérieure Sakra lui aurait procurées. C'est de cet arrière-plan associant la royauté birmane au prosélytisme bouddhique que s'inspirent les cérémonies de Thibaw et de Inlé, avec l'emploi notamment de véhicules processionnels identiques à ceux utilisés lors des parades royales comme lors des processions bouddhiques en milieu birman ${ }^{16}$, avec l'usage également d'attributs royaux lors du transport des images de Bouddha, ou encore avec le rappel constant d'un fonds légendaire lié aux pérégrinations du Bouddha et du roi Alaungsithu dans les régions périphériques de montagne, au nombre desquelles, affirment les chroniques locales, celles de Inlé et de Thibaw.

La parade du Kandawkyi met également en scène des courses de pirogues dont elle consacre la métamorphose. Comme partout ailleurs en Asie du Sud-Est bouddhique, les courses accompagnent traditionnellement les migrations des näga et, avec elles, les mouvements de flux et de reflux des eaux dont dépendent les récoltes (Archaimbault, 1972). Appelées "tête du nāga», les échancrures qui décorent la proue des pirogues intha en constituent un lointain rappel. Ces longues pirogues très caractéristiques, sur lesquelles plusieurs dizaines de rameurs peuvent prendre place, sont habituellement rangées sous les pilotis des monastères lacustres. Lors de la procession annuelle d'Inlé, elles entrainent l'embarcation sacrée où reposent les quatre images de Bouddha; elles sont également utilisées pour les courses qui ponctuent la cérémonie processionnelle, par référence à la compétition ayant opposé la divinité Sakra au roi birman Alaungsithu dans sa quête impossible du Mont Meru ${ }^{17}$. Mais, à Inlé comme ailleurs, les courses de pirogues sont désormais limitées à leur plus simple expression afin, selon les sources officielles, de limiter la portée des paris qu'elles occasionnaient; les jeux de hasard n'en continuent pas moins de faire la renommée de la cérémonie de Bokyaw à Thibaw. La réapparition

16. Lors d'une prise de robe, de funérailles de bonze, ou à l'occasion de l'installation rituelle d'une ombrelle terminale sur une pagode, l'usage d'un karaweik (karawika en translittération) est à ma connaissance limité au milieu birman - influencé par les Môns - le long de la vallée de l'Irrawaddy et du delta.

17. La chronique de Phaung Daw U à Inlé fait longuement référence à la compétition qui aurait opposé la divinité supérieure Sakra (donnée comme l'équivalent bouddhique d'Indra dans le panthéon hindou) au roi birman Alaungsithu. Après avoir pris l'apparence d'un vieillard, Sakra défia Alaungsithu: la mauvaise barque du vieillard l'ayant emporté sur l'embarcation à figure d'oiseau mythique du roi birman, ce dernier se résolut à ne plus chercher le Mont Meru pour se consacrer exclusivement au prosélytisme bouddhique. Sakra lui aurait alors remis le bois précieux dans lequel le roi birman aurait taillé plusieurs images du Bouddha de dimensions réduites, au nombre desquelles celles emportées lors de la cérémonie processionnelle; c'est par référence à cet épisode qu'est parfois attribuée l'origine des courses de pirogues. Pour plus de détails voir Robinne 2000a, 265-267 et 278 . 
les embarcations royales étaient autrefois dessinées, c'est un karaweik - de bambou puis de métal - que représentent les embarcations transportant les images d'Inlé (et de Thibaw avant que la cérémonie processionnelle ne redevienne une fête de pagode), et c'est encore ce même oiseau mythique qui est reproduit en première page du programme officiel de la parade Kandawkyi (fig. 5). La fabrication des pirogues qui défilent lors de cette parade témoigne toujours de la dextérité des charpentiers birmans (fig. 6); la plupart n'en sont pas moins une pâle reproduction des embarcations traditionnelles: un manuscrit de l'époque Konbaung, conservé dans la bibliothèque du département d'archéologie à Rangoun, en fournit de nombreuses reproductions en couleur très détaillées ( $\mathrm{Rb}-1$ et fig. 7).

Ces influences multiples sont toutes issues du patrimoine birman dans ce qu'il peut avoir de plus universel. Malgré tous les artifices, la parade du lac Kandawkyi se limite essentiellement à une forme sécularisée, à dominante corporatiste, des processions royales et des cérémonies de Thibaw et d'Inlé. Placée sous le haut patronage du « Secrétaire 1 » d'alors, elle a officiellement pour raison d'être: « Le renouveau de l'esprit patriotique et la préservation de l'héritage culturel et de l'identité nationale » (Khin Maung Nyunt 1996,59). Dans sa composition, elle se veut à l'image du découpage administratif du pays, doublée d'une représentation des principales corporations dépendant des différents ministères (Rb-5):

- En tête du cortège : l'embarcation officielle du comité organisateur encadrée de quatre autres embarcations à figure d'animaux mythiques tirés du panthéon bouddhique, reproduisant ainsi le modèle paradigmatique de quatre points autour d'un centre de la cosmogonie bouddhique appliqué au pouvoir central;

- Puis viennent les embarcations symbolisant les sept États périphériques auxquels les Constitutions de 1947 et de 1974 ont donné une dimension ethnique (États arakanais, chin, kachin, shan, kayah, karen,

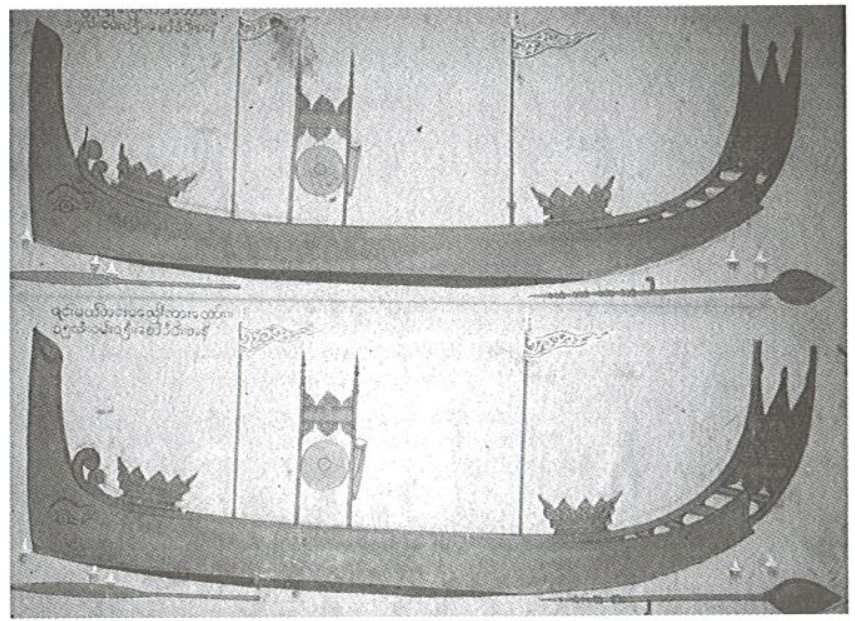

Fig. 7. Manuscrit de l'époque konbaung représentant les pirogues de parade royale de 1874 (document conservé au département d'archéologie de Rangoun) 
ministères et les diverses administrations y sont représentés - quand son référent est la royauté birmane, de nature ethnique quand son référent est le processus de birmanisation appelé « identité nationale », de nature laïque quand son référent est le bouddhisme via les cérémonies processionnelles. En aucun cas la parade folklorique du Kandawkyi n'a pu pénétrer les systèmes de représentations auxquels elle était pourtant censée s'identifier.

En revanche, considérée du point de vue de la neutralisation de la composante ethnique qui est l'un des objectifs de la junte, le processus de captation des cérémonies processionnelles d'Inlé et de Thibaw par le biais de la parade Kandawkyi à Rangoun peut être considéré comme un succès. Si les deux rituels avaient en commun d'être inclusifs, au sens où ils constituaient le vecteur du lien communautaire dans un processus de hiérarchisation des relations interethniques, le dédoublement du religieux et du politique a soustrait cette part de dynamique contenue dans les cultes locaux. Ils contrastent en cela avec la parade Kandawkyi, qui avait pour caractéristique première d'être exclusive, la raison sans doute pour laquelle il y fut mis un terme.

Dans sa tentative de captation de rituels locaux, la junte birmane n'a fait que détourner les cérémonies d'Inlé et de Thibaw de leur contenu politique afin de s'en approprier les retombées. La sédentarisation du rituel de Thibaw et l'excroissance du rituel d'Inlé sont les phases opposées d'un processus de birmanisation tourné vers les mêmes enjeux. Les configurations extrêmes données aux deux rituels contribuent en effet à la neutralisation du bouddhisme dans sa fonction d'opérateur commun, vecteur en tant que tel de la gestion sociale du territoire dans un contexte multiethnique. Le mouvement d'oscillation entre les pouvoirs régionaux, c'est-à-dire de minorités à minorités entre lesquelles le bouddhisme faisait office d'opérateur par le biais des rituels processionnels, est désormais sous le contrôle du seul pouvoir central. La parade Kandawkyi en fut le pur produit : elle constitua le prolongement immédiat d'un processus d'appropriation des rituels locaux auxquels était donnée une apparence sécularisée, et elle concentra, sous une forme corporatiste, la dimension politique dont les cérémonies processionnelles ont localement été dépossédées. Si le politique constitua la toile de fond de la parade Kandawkyi, elle ne bénéficia jamais, malgré tous les artifices, du cadre spirituel que lui auraient procuré les images d'Inlé ou de Thibaw. Le pouvoir central n'en met pas moins en scène ses rois et son panthéon bouddhique pour mieux se donner à voir. Et il puise dans le fonds local qu'il neutralise la légitimité dans laquelle il s’imagine. 
MILnE, Leslie

1924 - The Home of an Eastern Clan. A Study of the Palaungs of the Shan State, Oxford, Clarendon Press, $428 \mathrm{p}$.

PILINSKY, Jos

2002 - « La région du Myaukkodaung (État de Momeik) », traduction d'un rapport adressé par le délégué Commissioner de Bhamo au Commissioner de la Division Nord de la Haute Birmanie, Annexe à la lettre de Rangoun du 20 juin 1890. Présentation par François Robinne, Aséanie 10, p. 143-165.

ROBINNE, François

1998 - « Les immigrés Intha, la transhumance des buffles et la circumnavigation bouddhique du lac Inlé (État shan de Birmanie) », Pierre Pichard et François Robinne (éd.), Études birmanes en hommage à Denise Bernot, Paris, EFEO, p. 333-370.

2000 a - Fils et maîtres du Lac. Relations interethniques dans l'État Shan de Birmanie, Paris, CNRS Éditions, $364 \mathrm{p}$.

$2000 \mathrm{~b}$ - «Emergence of a Leading Group: A Case Study of the Inter-Ethnic Relationships in the Southern Shan State », J. Michaud (ed.), Turbulent Times and Enduring Peoples. Mountain Minorities in the South-East Asian Massif, Richmond, Curzon Press, p. 151-163.

2007 - «Transethnic Social Space of Clans and Lineages. A Discussion of Leach's Concept of Common Ritual Language », François Robinne and Mandy Sadan (eds), Social Dynamics in the Highlands of Southeast Asia, Leiden, Brill, Handbook of Oriental Studies III (8), p. 283-297.

2008 - «Catégories ethniques versus réseaux transethniques. Critique du concept d'ethnicité appliqué au paysage birman », Gabriel Defert (sous la direction de), Birmanie contemporaine, Paris, Bangkok, Les Indes savantes / IRASEC, p. 123-142.

ROBINNE, François and SADAN, Mandy

2007 - «Postscript: Reconsidering the Dynamics of Ethnicity through Foucault's Concept of 'Spaces of Dispersion' ", François Robinne and Mandy Sadan (eds), Social Dynamics in the Highlands of Southeast Asia, Leiden, Brill, Handbook of Oriental Studies III (8), p. 299-308.

SCOTT, J. George and HARDIMAN, J. P.

1901 - Gazetteer of Upper Burma and the Shan States, Rangoon, Superintendent Government Printing, Part II, vol. 1.

The New Light of Myanmar

27 September 1994, « Myanma Traditional Regatta held in Pakokku ».

24 October 1994, "General Maung Aye visits Inlay Phaungdaw-U, tours eastern Shan State».

21 November 1996, « Chairman of State Law and Order Restoration Council General Tan Shwe observes opening pageantry and events of Seventh Cultural Regatta Festival».

\section{Références en birman}

Rb-1 Thwak tō mhu akham: anā:, cātwè 1, kañ: kyoñ: thwak tō mhu puṃ [Expéditions royales, partie I: expéditions par voies de mer et par voies d'eau], s. d. 